\title{
Planificación urbana y regional para Santiago de Chile desde 1960: aporte conceptual y proyectual temprano para la sustentabilidad
}

\section{Urban and regional planning for Santiago de Chile since 1960 and pro- jecting early conceptual contribution to sustainability}

\author{
María Isabel Pavez Reyes \\ Facultad de Arquitectura y Urbanismo de la Universidad de Chile \\ Portugal 84, Santiago de Chile \\ Email: mpavez@uchilefau.cl
}

\begin{abstract}
RESUMEN
Se confronta algunos aspectos relevantes en los proyectos aprobados oficialmente para la ciudad y micro-región de Santiago desde 1960, con los correspondientes conceptos hoy explicitados y globalizados en materia de sustentabilidad. Se intenta superar los reduccionismos presentes en los pocos y breves análisis de los últimos años al respecto, y valorar más equilibradamente una experiencia relevante del Estado de Chile, en los momentos en que es necesaria una participación mejor informada en las discusiones actuales para una "nueva planificación"de los asentamientos humanos y territorios habitables. Se concluye que, a pesar de las limitaciones metodológicas y de recursos de información en la época de su formulación, los cuerpos conceptuales aplicados en dicha planificación, representan una etapa temprana de un discurso hoy maduro sobre la sustentabilidad.
\end{abstract}

Palabras clave: Planificación urbana y regional, ordenamiento territorial, Santiago de Chile, sustentabilidad, corredores urbano-regionales,

\begin{abstract}
It confronts some important aspects in officially approved projects for the city and micro-region of Santiago since 1960, with the corresponding concepts explicit and globalized today in sustainability. It tries to overcome the reductionism found in the few brief analysis of recent years about it, and a more balanced assessment of relevant experience of Chile, at times the need for a more informed participation in ongoing discussions for a "new planning "of human settlements and liveable areas. We conclude that despite methodological limitations and information resources at the time of its formulation, the bodies charged in the conceptual planning, represent an early stage of a-mature discourse about sustainability today.
\end{abstract}

Keywords: Urban and regional planning, land use, Santiago de Chile, sustainability, urban-regional corridors. 


\section{INTRODUCCIÓN}

Los pocos análisis habidos hasta ahora sobre las prácticas en Planificación Urbana y Regional por el Estado de Chile en su etapa madura (1950-1979), fijaron la atención en sólo parte de los proyectos y realizaciones y con referencia a planos, desestimando las memorias complementarias asociadas; además, los planos considerados no siempre han correspondido a los proyectos originales. Por otra parte, se ha acoplado la obra concreta de la Planificación Urbana y Regional chilena del período citado, y a sus autores, a los principios doctrinarios del Movimiento Moderno en Urbanismo - Carta de Atenas (LE CORBUSIER, 1942 [Ed. 1971/73])- y por esta vía, a un "urbanismo utópico", fácilmente asociable, a su vez, a la imagen del "Estado Planificador", también considerado utópico. Adicionalmente, se ha afirmado que los planificadores de la época sólo se interesaron en producir una "imagen física-objetivo" en sus proposiciones, y se han soslayado los problemas derivados de la pérdida de objetivos mayores en los equipos que se fueron sucediendo a través del tiempo, de los traspasos de responsabilidades desde el Ministerio de Obras Públicas al Ministerio de la Vivienda y Urbanismo (1965) para obras de largo plazo, y de la evolución no siempre positiva de las instituciones responsables. Por último, en ocasiones los medios producidos no fueron observados como parte de un sistema originalmente proyectado con elementos componentes articulados, sino como un conjunto vago de objetos sueltos (PAVEZ, 2006).

Todo lo señalado ha dado como resultado afirmaciones sin fundamento, representando reduccionismos que es necesario superar. Se intenta pues, valorar más equilibradamente una experiencia relevante del Estado planificador urbano y regional chileno, en los momentos en que es necesaria una participación mejor informada en las discusiones actuales para una "nueva planificación"de los asentamientos humanos y territorios habitables. Para efectos de la confrontación propuesta se consideró:

- un conjunto de los conceptos contemporáneos de sostenibilidad, en diversos autores e instituciones (FOLCH, 2003; PESCI, 2002; ROMERO, 2004; GORE \& UNIVERSIDAD DE CHILE, 2002);

- la información en los planos y memorias correspondientes a la aprobación oficial (1960) de los proyectos coordinados: "Plano Intercomunal de Santiago"y "Plano Microregional - Región IV - B Santiago de Chile" (Ministerio de Obras Públicas de Chile, 1960), (PARROCHIA, et al., 1994);

- las específicas medidas para la protección de los corredores ecológicos y biológicos de Santiago de primera magnitud (piemonte andino metropolitano, corredor fluvial y recreacional del Mapocho);

- las específicas medidas para el incremento y diversificación funcional de las teselas verdes urbanas y su conexión entre ellas y con la matriz agrícola.

De esta forma, se observó el grado de la consideración por la planificación de interés, de algunas de las restricciones que imponía la complejidad propia del espacio geográfico intervenido (PAVEZ et al., 2009-2010).

1. Los conceptos en la formación de los autores. Las acciones básicas previas a la proposición de los Planes Intercomunal y Microregional de Santiago de Chile. Los objetivos de los planes coordinados y el proyecto urbano como parte de un sistema territorial

\subsection{Los conceptos en la formación de los autores}

Se observará que la formación de los autores de los planes coordinados aprobados 
desde 1960, Intercomunal y Microregional de Santiago, en especial del arquitecto y urbanista Juan Parrochia Baguin (n.1930) -Premio Nacional de Urbanismo 1996-, en la entonces "Facultad de Arquitectura"de la Universidad de Chile (1947 a 1952), y posteriormente en otras instituciones en el extranjero, comprende una fuerte influencia de las escuelas del biólogo y sociólogo escocés Patrick Geddes (1854-1932); del historiador norteamericano de la civilización Lewis Munford (1895-1990) -discípulo de P. Geddes, y fundador de la Regional Planning Association of America (1923)-; del arquitecto y urbanista francés Robert Auzelle (1913-1983) -fundador y director del Centro de Estudios (1948) al origen del Centre de Recherche d'Urbanisme de la Dirección General de Urbanismo y la Habitación, convertida en 1949 en Dirección del Ordenamiento Territorial-; del arquitecto y urbanista francés Gaston Bardet (19071989) -único representante del "Urbanismo Culturalista"en su país y principal opositor al urbanismo del Movimiento Moderno(PAVEZ, 2006). Constatamos, también, la impronta en J. Parrochia B. del cuerpo de ciencia elaborado por su profesor, el precursor chileno del ordenamiento territorial con base geográfica, desde los años 1930, arquitecto y urbanista Luis Muñoz Maluschka (1896-1974), comprendiendo transferencias culturales desde Alemania en virtud de su interlocución entre c. 1928 y 1953 con el geólogo, paleontólogo y geógrafo alemán Kurt Brüning (1897-1961, fundador en 1947 de la Academia de Investigación del Espacio y Planificación Territorial, Akademie für Raumforschung und Landesplanung (ARL, existente hasta ahora en Hannover), entre otras personalidades afines (PAVEZ, 2006).

La aplicación del método del "Regional Survey", propuesto por Geddes (influenciado, a su vez, por los geógrafos franceses y la sociología de Le Play), a partir del cual no era suficiente limitarse al análisis de la ciudad, sino que era necesario analizar toda la región en la cual estaba la entidad urbana, a la vez que considerar todos los puntos de vista posible, tanto el sociológico, como el geográfico, el histórico y el económico (1907), es sin duda un aspecto especialmente considerado por los autores de la planificación de 1960. Bardet por su parte, llegó a indicar la obsolescencia del término tradicional de Urbanismo, toda vez que los problemas del asentamiento humano están en todo el territorio habitable. De la región provienen los materiales utilizados en su estructura física, de ella provienen los abastecimientos para la vida de sus habitantes y ella le proporciona el marco de su paisaje caracterizador. Auzelle destaca la imperiosa necesidad de abandonar las improvisaciones en materia de ordenamiento territorial y de coordinar las diversas formas de ocupación en todo el territorio habitable, con especial atención a la ecología del paisaje. En Chile, Muñoz Maluschka consideró la Planificación Regional como la estructuradora básica de todo proceso de Planificación Territorial en el país, siendo autor de la teoría "Concepción dinámica del Urbanismo. Espacios vitales urbanos"(PAVEZ, 2006).

\subsection{Las acciones básicas previas a la pro- posición de los Planes Intercomunal y Microregional de Santiago de Chile}

En 1953 el Arqto. Luis Muñoz Maluschka, Jefe de la Sección de Urbanismo en la Dirección General de Obras Públicas de Chile (entre c. 1936 y 1953), basándose en las experiencias del Estado planificador de Baja Sajonia, logró el reconocimiento legal de los niveles territoriales de la planificación sobre el nivel de Comuna. Simultáneamente, logró la creación de la Dirección de Planeamiento en el Ministerio de Obras Públicas, la que debía abordar el estudio de los planes nacionales, regionales e intercomunales de ordenamiento territorial, además de la autorización técnica de los planos reguladores urbanos de nivel comunal. El instrumento "Plan Regulador Intercomunal"se creó como unidad compacta o como sistema satelitario. Otra novedad técnica fue la del Plan Director Territorial, con el objetivo de 
delimitar los Planes Regionales. Estos últimos delimitando los sistemas de núcleos poblados (CHILE - MOP, 1958).

El estudio, avance y revisión de los diversos planes concebidos en forma coordinada sería, en adelante, un principio fundamental especialmente para el manejo del territorio de las regiones urbanas. A partir de este momento, la acción urbanística se desarrollaría sobre todo el conjunto de las regiones metropolitanas emergentes - hasta seis en Chile-, según Muñoz (PAVEZ, 1993), coordinando sus escalones territoriales, los diversos tipos de asentamientos humanos urbanos y rurales con sus modos de vida, aplicando valores universales en la búsqueda del bien común.

Una de las obligaciones legales con la que comenzó sus labores la Dirección de Planeamiento del Ministerio de Obras Públicas, fue el estudio de una "división del territorio nacional en regiones con fines de planeamiento y coordinación". Las “unidades de planeamiento territorial"fueron concebidas con la participación de arquitectos, ingenieros, geógrafos y otros especialistas, en un esfuerzo de primera aproximación.
Se promovió también una política nacional tendiente a un mejor aprovechamiento del suelo urbano, suburbano, y rural, con el objetivo de un despliegue más racional de los servicios de urbanización, de energía, transportes colectivos y otros.

La división regional de Chile propuesta por la Dirección de Planeamiento de acuerdo a los Decretos Leyes $\mathrm{N}^{\circ} 150$, de 4 de julio de 1953, y No224, de 22 de julio de 1953, fue establecida oficialmente por Decreto Supremo N².004 de 28 de octubre de 1954.

Definían el territorio de una región las características geográficas (suelo, clima, demografía), las características geopolíticas (tensiones internas y externas), las características económicas (recursos de caza y pesca, minería, ganadería, riqueza forestal, industrial, comercio, actividades técnicas y profesionales, etc.), y las características sociológicas (equipamiento urbano y rural, estratificación social, nivel cultural, nivel educacional, etc.).

Se delimitaron, así, con iguales criterios, diez regiones para Chile:

REgiones DE CHILE - D.S. No 2.004 DE 1954

\begin{tabular}{|l|l|}
\hline REGIÓN I & Provincia de Tarapacá \\
\hline REGIÓN II & Provincia de Antofagasta \\
\hline REGIÓN III & Provincias de Atacama y Coquimbo \\
\hline REGIÓN IV & Provincias de Aconcagua, Valparaíso y Santiago \\
\hline REGIÓN V & Provincias de O'Higgins, Colchagua, Curicó, Talca y Linares \\
\hline REGIÓN VI & Provincias de Nuble, Concepción, Arauco y Bío-Bío \\
\hline REGIÓN VII & Provincias de Malleco, Cautin y Valdivia \\
\hline REGIÓN VIII & Provincias de Osorno, Llanquihue, Chiloé y Aysén \\
\hline REGIÓN IX & Provincia de Magallanes \\
\hline REGIÓN X & Territorio antártico chileno \\
\hline & \\
\hline
\end{tabular}

Cada una de las regiones se dividió en "microregiones", considerando los antecedentes de población, equipamiento, producción y relaciones. Cada microregión fue designada con el nombre de su ciudad capital, la que fue elegida, a su vez, de acuerdo a su jerarquía en cuanto a población y equipamientos. Para esto se exigió un distanciamiento espacial pruden- 
te entre ciudades de la misma categoría. Lo contrario obligó a fusionarlas, como por ejemplo en los casos de Valparaíso-Viña del Mar, o San Felipe-Los Andes.

Los arquitectos Amador Brieva Alvarado y Ventura González Montecinos, habían sistematizado un cuerpo teórico para efectos de dicha subdivisión, resultando el método propuesto de clara aplicación, obteniéndose pronto la certeza de su efectividad (BRIEVA \& GONZÁLEZ, 1958). Las relaciones interurbanas creaban lo que sus autores denominaron "sistema natural gravitacional entre núcleos poblados". La diversidad de núcleos poblados con diferencias en su número de habitantes, en sus servicios de utilidad pública, en sus posibilidades de conexión, de su área de explotación de la cual depende su subsistencia, etc., indicaba una necesaria colaboración de unas con otras, definiéndose con el tiempo una jerarquización.

Se consideró indispensable medir para cada núcleo poblado, su poder de atracción de otros núcleos y viceversa, y en qué grado se ejercían estas atracciones. La resolución de estas cuestiones fundamentales permitió definir e individualizar las unidades "micro-regionales". Los Planes Micro-Regionales se establecieron, así, como subsistemas de los estudios regionales.

Se observa que se definió una "Región Central" (Provincias de Aconcagua, Valparaíso y Santiago), con las cuencas del Maipo-Mapo- cho y del Aconcagua. Esta Región, a la que se le asignó el número IV, se dividió en las sub-regiones: IV-A: Valparaíso y el Río Aconcagua (Cuenca del Aconcagua), y IV-B: San Antonio, Melipilla y Santiago (Cuenca del Maipo-Mapocho), incluyendo las Micro-Regiones de Santiago y de San Antonio.

En la Región IV se observó un conjunto de valles menores junto al llamado "gran valle central"'(depresión central de origen tectónico), con capas vegetales que permitirían el desarrollo de cultivo intensivo de la vid, frutales y hortalizas. Las grandes planicies se apreciaban como propicias para el desarrollo de las poblaciones. La zona cordillerana que circunda la región, con grandes macizos y las mayores alturas del continente, con carácter minero, apropiada para el desarrollo de la industria y la energía eléctrica. La Cordillera de la Costa, más baja, con algunos pasos relativamente fáciles para la movilización. Una costa amplia, con playas alargadas y limpias, esencialmente turísticas, y establecimientos hoteleros modernos. En cuanto al clima, la Región IV presentaba estaciones bien demarcadas, con heladas en el comienzo y término del invierno. La permeabilidad del suelo y subsuelo y su estructura en general, era apropiada para el escurrimiento de las aguas, disminuyendo la humedad (KRAUSE, 1958).

Para la IV Región, la Dirección de Planeamiento estableció seis microregiones:

\begin{tabular}{|l|c|}
\hline \multicolumn{1}{|c|}{\begin{tabular}{|c|} 
MICROREGIONES DE LA REGIÓN IV Y HABITANTES, \\
MICROREGIONES REGION IV AND INHABITANTS,
\end{tabular}} \\
\hline Microregiones & Población (1952) \\
\hline La Ligua & 30.342 \\
\hline San Felipe-Los Andes & 105.234 \\
\hline La Calera-Quillota & 90.172 \\
\hline Valparaíso-Viña del Mar & 396.577 \\
\hline Santiago & 1.651 .796 \\
\hline San Antonio-Melipilla & 107.465 \\
\hline TOTAL & $\mathbf{2 . 3 8 1 5 8 6}$ \\
\hline
\end{tabular}


La Dirección de Planeamiento denominó a toda esta Región IV, "Región Metropolitana"(32.444 Km²). Su población total en 1952 era de 2.381 .586 habitantes, aproximadamente el $30 \%$ de la población de Chile.

De igual forma se estudiaron en 1953-54, todas las microregiones del territorio nacional, con criterios uniformes, configurándose en forma equivalente - sobre una base económica, técnica y social-.

El tercer escalón de la graduación creada fue el "conjunto intercomunal", apreciado como una unidad territorial mixta, urbana y rural, en la cual se debería integrar armónicamente las funciones de varias unidades urbanas, entre las cuales destaca una, por su mayor población mejor equipamiento $\mathrm{y}$ mayor producción pasando a constituir el centro comercial, social y cultural del conjunto. Más tarde se crearon los "Planes Seccionales", como sub-sistemas de los Planes Intercomunales y Comunales.

Cabe destacar que hacia fines de los años 1950 había ido tomando importancia la investigación geográfica y geomorfológica del área denominada Cuenca de Santiago. Después de sistematizarse y delinearse en un croquis morfológico los rasgos principales de la cuenca (BRÜGGEN, 1929), diversos autores enriquecieron y ponderaron su descripción e interpretación. Sin embargo, al comenzar la década de 1960, ningún autor había señalado en forma nítida los limites del "valle de Santiago", y se podía concluir que el conocimiento de esta microregión era muy precario (FLORES, 1966). Los estudios para un mejor conocimiento de la cuenca de Santiago realizados a mediados del siglo XX y principios de los años 1960 , incidieron especialmente en la búsqueda de agua subterránea para el abastecimiento de Santiago. Se estimaba en la época que si se quería adecuar las necesidades de una población en crecimiento a las disponibilidades hídricas, edáficas y otras que la cuenca presentaba, eran necesarios detallados estu- dios de geografía humana que justificaran nuevos estudios físicos para su mejor conocimiento.

La microregión de Santiago quedó señalada entonces, entre el cordón de Chacabuco y Angostura de Paine y las dos cordilleras, sistema de relieve que ocupa el $70 \%$ de la superficie, y que, al circundar completamente la planicie (cuenca de Santiago) formada por los rellenos de los ríos Maipo y Mapocho, dificulta las comunicaciones con el resto del país.

Hacia 1958 se constataba que el 31,5\% de los habitantes activos de la microregión de Santiago originaba el $65 \%$ de toda la producción industrial nacional; se estimaba que el rápido auge de la industria en esta microregión no había dado lugar a una oportuna racionalización de la ubicación de las industrias; así, la mayor parte estaba en la ciudad de Santiago y comunas adyacentes, en lugar de haberse ubicado en la periferia de la microregión, acercándose a los lugares de origen de las materias primas (Norte Chico, costa y sur del país), donde hubiera recursos de agua suficientes y donde se pudiera aumentar el área de explotación agrícola con obras de regadío y drenajes (KRAUSE, 1958). Los lineamientos del Ministerio de Obras Públicas en la época, señalaban posibilidades en Colina, Batuco, Curacaví, Paine, Buin y El Monte, unidades que pasarían a constituir centros industriales satélites de la capital, ubicados a no más de $60 \mathrm{Km}$ de distancia. Esto permitiría, en parte, la desconcentración de la población de Santiago, además de contribuir a elevar el estándar de vida de los sectores atrasados.

Quienes explotaban al máximo sus propiedades agrícolas estaban unidos a la capital por buenas vías de comunicación, y estas relaciones directas habían influido notablemente al estancamiento observado en el equipamiento urbano de los centros poblados del área, principalmente cabeceras comunales. En estos centros había un grave déficit en alcantarillado y agua potable, con el con- 
secuente bajo estándar sanitario en ellos. El $18 \%$ de la población de la microregión vivía fuera de Santiago (298.396 habitantes).

Por otra parte, se observaba una disminución de las relaciones entre los antiguos centros poblados existentes en la microregión, por las facilidades que iba presentando el acceso a Santiago.

El mayor transporte de carga se expresaba entre Santiago y el sur, por los productos transportados desde la próxima ciudad de Rancagua, y el sur más lejano del país para completar el abastecimiento de Santiago; también destacaba el camino a la costa, vía Padre Hurtado. La red ferroviaria mostraba una mayor relación con Valparaíso vía Llay Llay - La Calera, y con el puerto de San Antonio, vía Talagante. En cuanto al movimiento comercial aéreo en 1952, realizado por Los Cerrillos -el aeropuerto internacional de la época-, mostraba mayor movimiento hacia el norte y hacia la República Argentina que hacia el sur del país.

No obstante las carencias de información señaladas, los planificadores urbanos y territoriales de la época fueron conscientes de la inmensa riqueza natural de la cuenca del Maipo donde se implanta la ciudad de Santiago: sus ricos suelos; su abundante agua nivosa; su privilegiado clima que permite varias cosechas en un mismo año normal; sus hermosos micro y macro paisajes; su cercanía a las playas del litoral, con más de 300 kilómetros de extensión; su proximidad a los grandes centros de deportes invernales; su fácil acceso a los territorios más ricos y poblados de la República Argentina; su clara ubicación al centro de la larga faja del país y su relación directa con los tres puertos más grandes e importantes de Chile (Ventanas, Valparaíso y San Antonio) ubicadós frente a una rica reserva marina, animal, vegetal y mineral; su estratégica ubicación en el centro del eje oriente-poniente del océano Pacífico Sur; su rica área suburbana rural, con largas tradiciones históricas; sus pintorescos valles de quebradas y esteros, tanto en la Cordillera de los Andes como de la Cordillera de la Costa; su riqueza minera que rodea la ciudad con sus grandes mantos cupríferos, de cal y de áridos. Santiago se presentaba, además, como la encrucijada de grandes corredores de transporte hacia el sur, norte, oriente y poniente; tanto nacionales como internacionales (PARROCHIA et al., 1994).

\subsection{Los objetivos de los planes coordina- dos y el proyecto urbano como parte de un sistema territorial}

Al procederse a los estudios del Plan Intercomunal y del Plan Regional de Santiago (Arqto. Urbanista Juan Parrochia B., Arqto. Jefe de Intercomunales Juan Honold D.), se consideró la participación de profesionales de todos los servicios y de todas las disciplinas en la Dirección de Planeamiento y Urbanismo del Ministerio de Obras Públicas, en conjunto con todos los municipios comprometidos. La Universidad de Chile colaboró también auspiciando un amplio seminario, del "Gran Santiago", que produjo una voluminosa publicación de antecedentes de gran valor en 1958 (PARROCHIA, 1979).

Destacaron en la década de los años 1950 los estudios de los arquitectos Amador Brieva y González antes citado, Juan Honold, y Pastor Correa; de los ingenieros Directores de Vialidad Pedro Álvarez y Eduardo Paredes, y de Planeamiento, Jorge Kelemen; también los estudios de los ingenieros agrónomos Sergio Tartakowsky y José Luis Pistono, y el ingeniero sanitario Mario Riquelme, entre muchos otros. Por otra parte, en las labores de planificación regional y sectorial destacó el ingeniero Jefe de Aeropuertos, Florencio Oyarzún, quien dirigió los estudios del sistema nacional de aeropuertos y los primeros proyectos para el aeropuerto de Pudahuel (PARROCHIA, 1979).

Se proyectó entonces un "Plan Regulador Intercomunal de Santiago"(colección de planos impresa en colores por el I.G.M.), con un horizonte de tiempo situado en el año 2000 y una hipótesis de población de 6 
millones de personas. Fue aprobado desde 1958 técnicamente y desde 1960 oficialmente, y coordinado con unos planes "Regulador Regional", y "Microregional"de Santiago (colección de planos impresa en colores por el I.G.M.), los que reconocieron y trataron el ámbito urbano como parte de un sistema territorial; fueron objetivos de estos planes (PARROCHIA et al., 1994):

- Proteger los recursos agrícolas y la ecología de la región, dedicando las áreas improductivas a la forestación, a parques regionales y asentamientos humanos, sacando el mejor partido de la hidrografía y de la orografía, reforestando todo el territorio entre la cota 700 y 1.000 de altura; transformar la agricultura tradicional - artesanal y extensiva - en intensiva, con objetivos industriales y de exportación, para competir con éxito en el mercado de suelos y, a la vez, producir nuevos bienes y recursos para el bienestar de la población, de la región y del país.

- Recuperar los ámbitos ecológicos de la vida silvestre en todos los cerros, montañas y mares de la región.

- Reforestar los terrenos de baja calidad agrícola, cuyos bosques fueron destruidos por el consumo indiscriminado de leña y carbón del siglo XIX y principios del siglo $\mathrm{XX}$, para contribuir a descontaminar la atmósfera sin perjuicio de los métodos físicos y químicos para evitar la producción de gases y polvos tóxicos.

- Formar núcleos urbanos de diversos tamaños y especialidades, distribuidos de acuerdo a los corredores de transporte de la región y sus recursos naturales, implantando un sistema de "ciudadesconstelación”, esto es, un conjunto de núcleos urbanos interrelacionados, de diferentes tamaños y luminosidades, manteniendo una autonomía en los aspectos básicos de la vida humana (habitación, trabajo, comercio, etc.), formando, entre todas, una capital multinucleada.

- Mantener la relación de todas las unidades separadas como las integradas (bloques intercomunales y sectores intercomunales) entre sí, sin interferencias ni obstáculos, pudiendo todas ellas, además, acceder al área rural en un corto tiempo.

- Complementar la red de transporte con sistemas de by-pass, permitiendo el paso de sur a norte y de oriente a poniente del territorio nacional, de todo tipo de vehículos y carga, sin pasar por la ciudad de Santiago.

- Impulsar la explotación de los recursos mineros de la región.

- Multiplicar los lugares y centros de esparcimiento en valles, lagos, lagunas, y alta montaña.

- Intensificar el uso de los recursos turísticos y recreacionales dentro de la región.

- Recuperar los suelos con malos drenajes y $\sin$ regadío, especialmente los de las zonas norte del valle de Santiago (Colina, Batuco, Peldehue), el valle de Curacaví y el valle de Casablanca.

- Orientar y guiar el crecimiento de los núcleos metropolitanos (Valparaíso y Santiago) de la Región IV de la época (Provincias de Aconcagua, Valparaíso y Santiago), para lograr una desconcentración dentro de esta. Propender al crecimiento urbano por:

a) renovación, rehabilitación y remodelación de los cascos antiguos de las ciudades grandes y pequeñas de la región,

b) por relleno de las áreas eriazas existentes dentro de dichas ciudades, y

c) por satelización, basada especialmente en las ciudades existentes, y 
también en algunas nuevas ciudades, donde el suelo fuera de baja calidad.

- Crear núcleos industriales especializados, desconcentrados adecuadamente dentro de la región, evitando la contaminación.

- Crear núcleos urbanos de calidad para aumentar la oferta de terrenos adecuados para la vida de los estratos socioeconómicos bajos, medios y altos, para el comercio, la industria y el esparcimiento dentro de la región de Santiago, evitándose con ello la especulación urbana.

- Producir un borde estructurado en el contacto entre las áreas urbanas y rurales, mediante zonas suburbanas, conteniendo equipamiento metropolitano, zonas de parcelas residenciales, de producción agrícola intensiva, y recreacionales.

Por sobre todo, se quiso mantener las formas urbanas incorporadas a la naturaleza en el diseño de los centros poblados, evitando la ciudad redioconcéntrica de crecimiento repetitivo, monótono y alejado de su cuadro natural (PARROCHIA et al., 1994).

En el caso de la Micro-Región de Santiago ("IV-B"de la época), un gran anfiteatro, producido por el inicio norte de la depresión central de Chile, en la cuenca media del río Maipo y conformado fundamentalmente de norte a sur, por los ríos Puangue, Lampa y Colina, en sentido norte - sur; el Huechuraba, el Mapocho y La Aguada, el propio río Maipo en sentido oriente - poniente, y el Clarillo, el Paine y el Angostura, en sentido sur a norte, fue el espacio base del planeamiento. Se conformó así, lo que el urbanista del plan, Arqto. Juan Parrochia, denominó una "pauta de desarrollo MicroRegional"sobre un esquema de cuatro ejes hidrográficos centrales, proyectados hacia el norte y el sur, por tres ejes verticales principales y tres ejes secundarios en cada una de esas direcciones (PARROCHIA et al., 1994).
En lo señalado específicamente para el espacio rural de la Micro-Región de Santiago, el plano correspondiente - a escala original 1:100.000- dio cuenta de la heterogeneidad espacial del territorio, distinguiendo, entre otras, teselas de recursos, teselas con limitaciones, teselas introducidas, corredores ecológicos y la matriz, que en este caso es agrícola:

"Todo cultivo"; "Riego limitado - posible todo cultivo"; "Riego limitado"; "Secano - posible todo cultivo"; "Secano - posible riego limitado"; Secano"; "Pantanoso recuperable"; "Pantanoso"; "Sin drenaje - recuperable total"; "Morrenas"; "Sin drenaje - recuperable parcial"; "Praderas y forestales"; "Áreas de forestación proyectadas" (PARROCHIA et al., 1994).

Por otra parte, en los Planes Regulador Intercomunal de Santiago y de la Micro- Región IV-B, el núcleo central de Santiago se irradia hacia el territorio regional y nacional por nuevos corredores asociados a "cuñas verdes", para lograr una comunicación y un transporte más rápido, eficiente y paisajísticamente más logrado. A la inversa, la naturaleza penetra hasta el centro de la ciudad mediante esas mismas "cuñas"-los cerros Manquehue, Bosque de Santiago, San Cristóbal, Renca y Colorado; los ríos Mapocho y Maipo, y el zanjón de la Aguada, tanto al occidente como al oriente-, o por accidentes artificiales, en base de equipamiento metropolitano -el fundo La Laguna y el gran Centro de Abastecimiento Agrícola Occidental, el Aeropuerto Los Cerrillos, el Campus La Castrina de la Universidad de Chile--.

La consideración del conjunto total del territorio llevó a la apreciación de la importancia de mantener y potenciar ciertos corredores ecológicos en el entorno de Santiago como se verá más adelante.

En la planificación para Santiago analizada, se programó un sistema de satélites (residenciales, agrícolas, industriales y de recreación), distribuidos dentro de la microregión metropolitana, en radios de 15, $30 \mathrm{y}$ 
45 kilómetros de distancia del núcleo principal, con diversos grados de comunicación y autonomía relativa.

Actualmente vemos que uno de los modelos de ordenamiento espacial urbano-regional apoyado por la mayoría de los urbanistas de la sostenibilidad es, precisamente, el de la desconcentración concentrada: se recobra la escala humana en núcleos urbanos de au- tonomía relativa, cercanos a la ciudad madre, disminuyéndose el consumo de energía por movimientos y transporte. El modelo aspira a la "región urbana"donde, en los intersticios de su multifocalidad, en el espacio "libre"-que no significa vacío, ni intactopueda aflorar la matriz, pero también ciertos tipos de uso: agrícola, forestal, hidrológico, etc., articulados entre sí (PESCI, 2002).

Fig. 1. "PLANO MICRO-REGIONAL, REGIÓN IV-B, santiago de chile", 1960.

Fig. 1. "MICRO-REGIONAL LEVEL, REGION IV-B, santiago de chile", 1960.

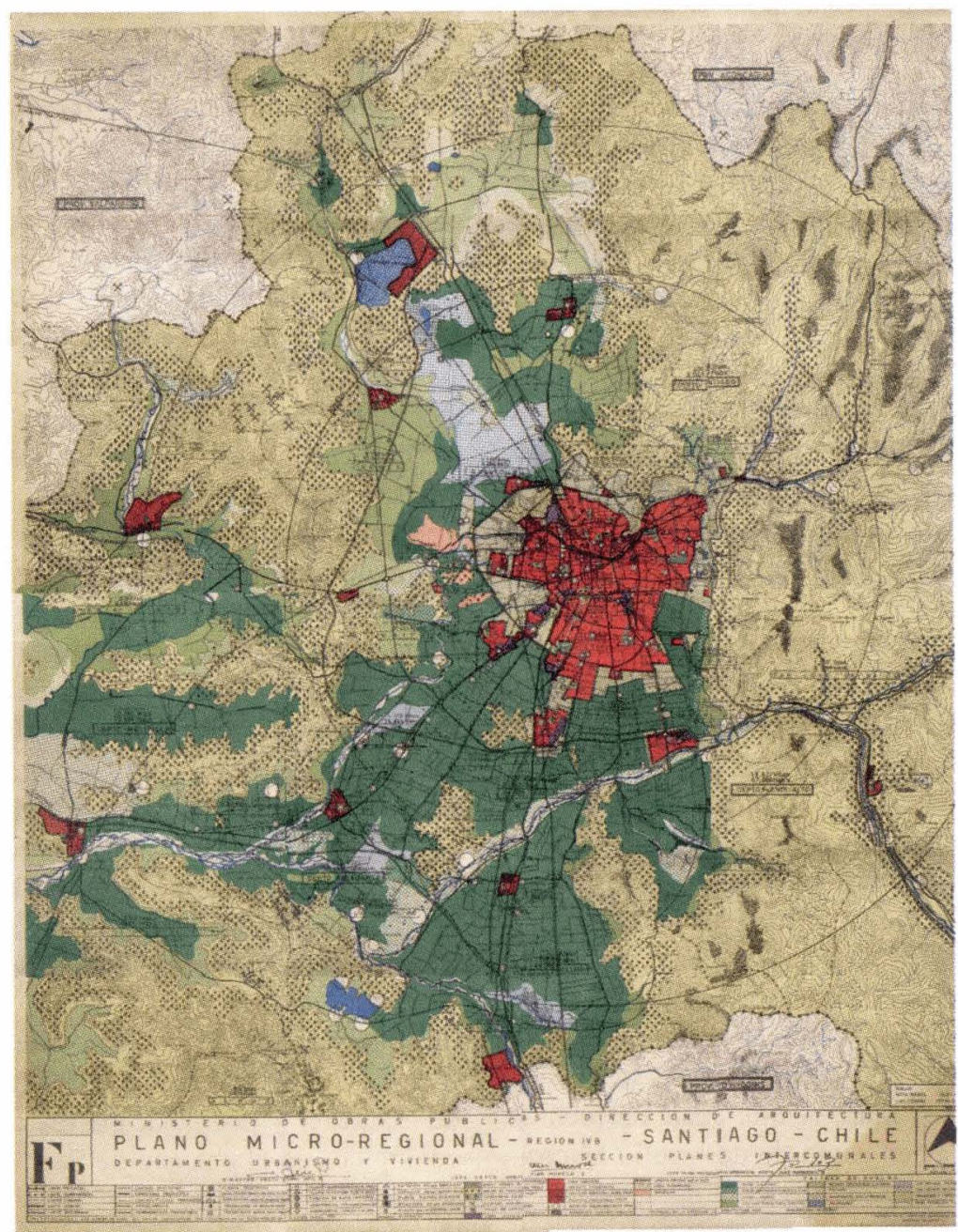

Fuente: CHILE - MINISTERIO DE OBRAS PÚBLICAS, Dirección de Arquitectura, D. Urbanismo y Vivienda, Sección Planes Intercomunales, "PLANO MICRO-REGIONAL - REGIÓN IV-B SANTIAGO DE CHILE". Firmado por Director Arqto. E. Weil W.; Jefe Depto. Arqto. J. Honold D.; Jefe Plan Intercomunal Urbanista Arqto. J. Parrochia B. (F-P; 96 x 76 cm; N²5567), escala gráfica. Reducción de escala original 1:100.000; Instituto Geográfico Militar, Santiago de Chile, s/f (1960). Colección de planos. [En Archivo Histórico Nacional, Colección "Juan Parrochia Beguin”]. 


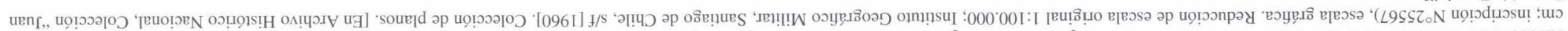

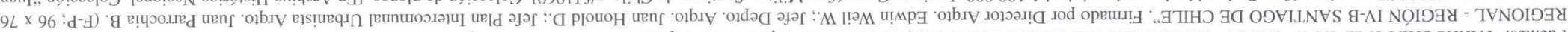

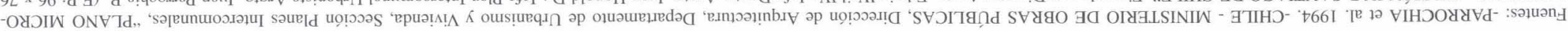

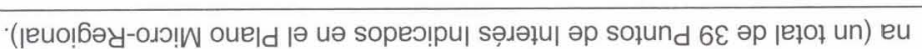

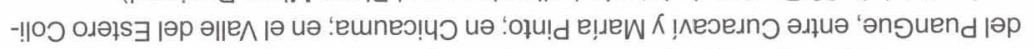

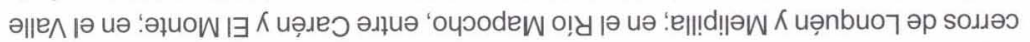

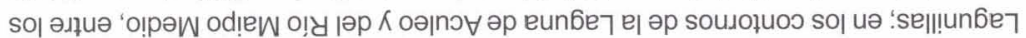

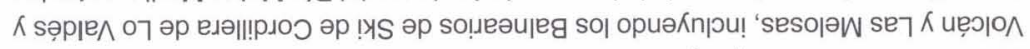

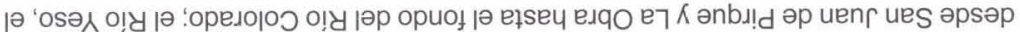

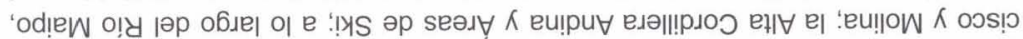

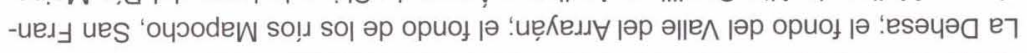

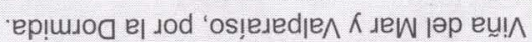

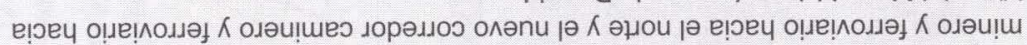

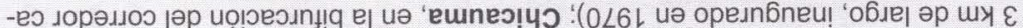

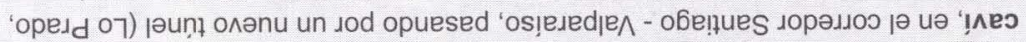

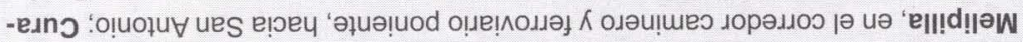

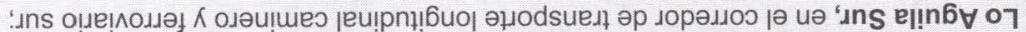

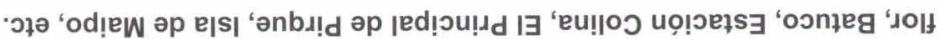

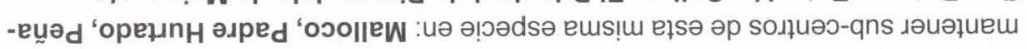

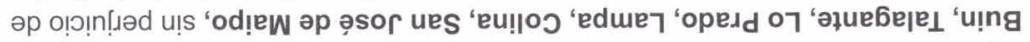

.

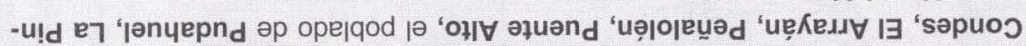

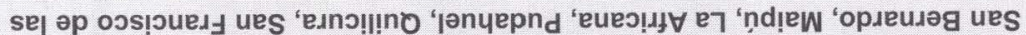

seiquon

sełs!̣uo!sınכx $\kappa$ səz

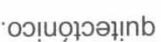

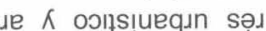
-ә孔u! 일 әр solunfiuos so||ə uә әsıе|еłsu! op -uәpnd 'sejoojube ou

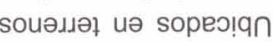

\section{obe!̣ues әp !!}

-odọ, -әр әр sәлорәлно sol

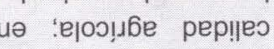
ереш әр sоиәдәә и suy strp oppowoud e! -uels! eun e sopes!̣n

sury

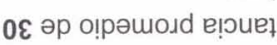
-s!̣p eun e sopeọqn

sury gle OL әр o!̣powoud e!̣uet -s!̣ eun e sopesian

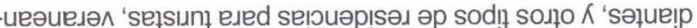

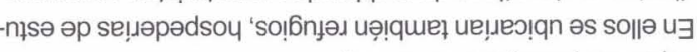

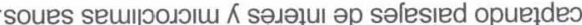

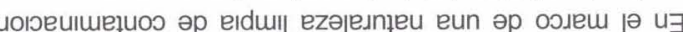
ене ерел

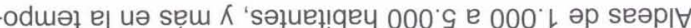
: SOIY $\forall \exists N 7 \forall 9$ S $\exists \perp I 7 \exists \perp \forall S$

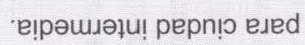

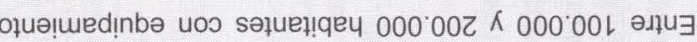

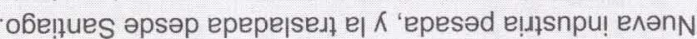
: $\unlhd \exists า \forall I Y \perp S \cap a N I$ S $\exists \perp I 7 \exists \exists \perp \forall S$

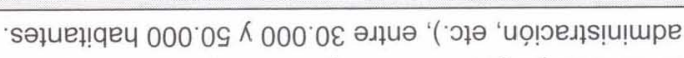

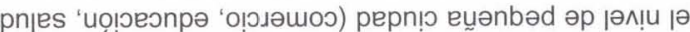

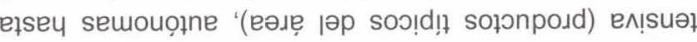

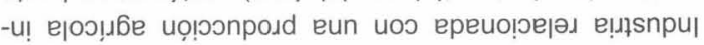

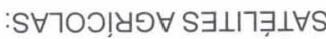

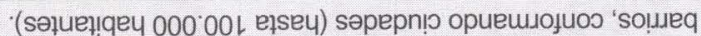

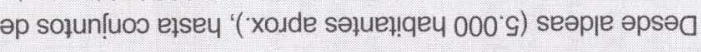
S $\exists 7 \forall I O N \exists \triangle I S \exists Y$ S $\exists \perp I 7 \exists \perp \forall S$

siodomew el ep

omuas ja epsap sury

upoesian

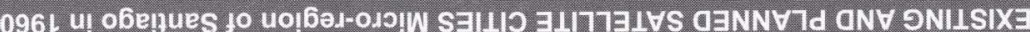

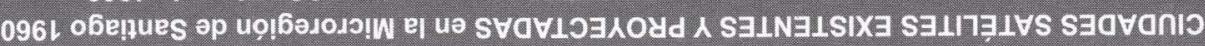


Fig. 2. "PLANO INTERCOMUNAL DE SANTIAGO", Plano: Vivienda - Área Suburbana Centros Cívicos - Vialidad - Industrias - Áreas verdes.

Fig. 2. "INTERCOMMUNAL SANTIAGO PLANO", Plan: Housing - Suburban Area - Civic Center - Highway - Industries - Green areas.

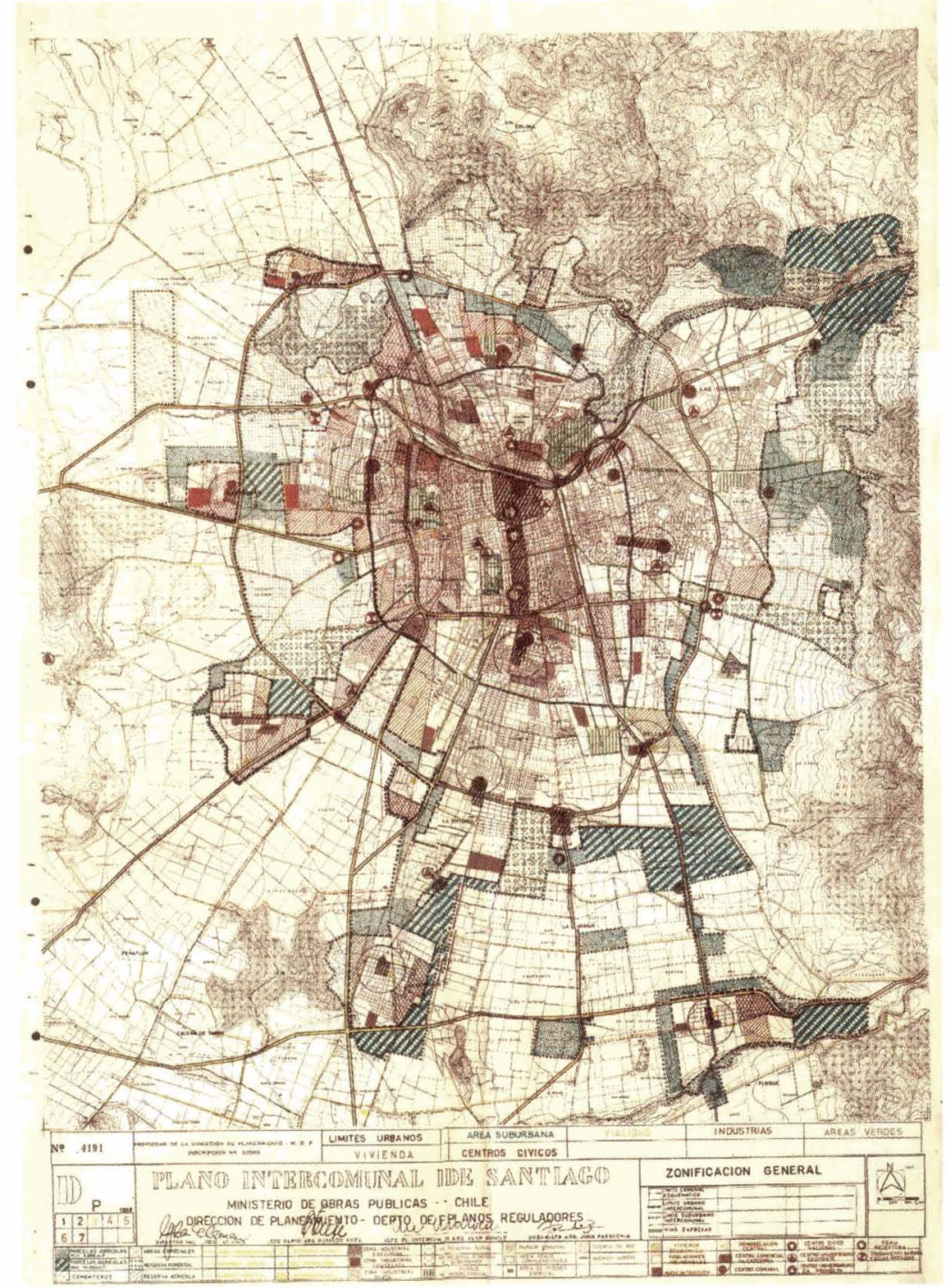

Fuente: CHILE -MINISTERIO DE OBRAS PÚBLICAS, Dirección de Planeamiento, D. Planos Reguladores, "PLANO INTERCOMUNAL DE SANTIAGO", firmado por Director Ing. J. Kelemen; Jefe Departamento Arqto. O. Hufe; Jefe Plan Intercomunal Arqto. J. Honold; Urbanista Arqto. J. Parrochia (Colección de planos de 77 x 55 cm.; Escala Gráfica (escala original 1:20.000), 1958, Instituto Geográfico Militar. [En Archivo Histórico Nacional, Colección "Juan Parrochia Beguin"].

Se asevera hoy nuevamente que el sistema urbano no puede desligarse del sistema territorial global en el que se encuentra inmerso (FOLCH, 2003), y que no habrá solución del territorio urbano sin articulación integral urbano-rural (PESCI, 2003).
Por otra parte, vemos que el Plano Intercomunal de Santiago fue trabajado a escala 1: 20.000 -escala microterritorial/ecológica, entre 1:10.000 y 25.000 (FOLCH, 2003)-, en tanto que el Plano Microregional de Santiago fue trabajado a la escala 1:100.000 
- escala mesoterritorial/paisajística, entre 1:25.000 y 100.000 (FOLCH, 2003). Cada ámbito escalar tiene asociado un tipo de fenómeno que se torna incomprensible contemplado desde demasiado cerca o desde demasiado lejos (FOLCH, 2003).

Ambos proyectos consideraron además un arco temporal que alcanzó al año 2000, considerando una proyección de población de 6 millones de habitantes. Corresponde ello a las actuaciones territoriales, las que deben considerar arcos temporales largos, o muy largos (FOLCH, 2003).

\section{Medidas para la protección de los corredores ecológicos y biológicos de primera magnitud: las riberas del río Mapocho y el piedemonte andino me- tropolitano}

El "Plano Microregional - Región IVB Santiago de Chile"1960, contempló un sistema de balnearios, parques y costaneras junto al río Mapocho, como parte del corredor metropolitano fundamental oriente - poniente desplegado entre la Cordillera de los Andes y la Cordillera de la Costa, con 100 km de largo y 350 metros de ancho aproximadamente.

Como consecuencia del trabajo coordinado realizado, el sistema propuesto aparece simultáneamente en el Plan Intercomunal de Santiago, desplegado a todo lo largo de río Mapocho urbano, entre los límites del área definida entonces como intercomunal ("Parque Intercomunal Cuenca del Mapocho", con 690 Hás.).

Destaca, además, la disposición de reservas forestales y agrícolas al oriente de la ciudad, en el piedemonte andino metropolitano, entre un "Límite Urbano Intercomunal"y un "Límite Suburbano", tanto para efectos de mantener áreas de absorción de flujos de agua $-y$, con ello, contribuir a evitar las inundaciones de la ciudad-, como para evitar los efectos severos sobre la calidad del aire, entre otros beneficios. Era este un corredor ecológico de $40 \mathrm{Km}$ de largo y 4 $\mathrm{km}$ de ancho aproximadamente, que nunca debía ser urbanizado, pero que sí podría generar proyectos de forestales y agrícolas.

Se establecierón "Reservas Forestales Rurales": esto es, áreas rurales inmediatas a la ciudad, destinadas a plantaciones de árboles y matorrales que se adaptaran a las condiciones naturales del terreno, y "Reservas Agrícolas y Forestales Suburbanas": terrenos agrícolas y ganaderos que debían conservar la utilización que presentaban en la época, y áreas destinadas a reservas forestales (distribuidas en superficies de 300 a 800 hectáreas).

Finalmente, todos los cerros que rodean Santiago quedaron bajo el estatus de Parque, constituyendo un cordón verde mayor para la recreación tanto de la población prevista en la microregión como en la metrópolis: cerro Alvarado (Las Condes); cerro El Observatorio (Las Condes); cerro Manquehue (Las Condes); cerro Colorado (Quilicura); cerro Renca (Quilicura); cerro Vizcacha (Puente Alto); cerro Negro (San Bernardo); cerro Chena (San Bernardo); cerro Blanco (Santiago).

En el caso de Santiago, dado que en las cordilleras se acumulan las mayores cantidades de lluvias y también las nieves y glaciares que producen las aguas que recargan los acuíferos, o bien que alimentan su escurrimiento superficial y subsuperficial a través de los ríos, quebradas y arroyos y de los acuíferos subterráneos, el almacenaje de agua en el subsuelo es un proceso fundamental para su subsistencia como sistema ambiental y como ciudad, tanto más cuanto que las condiciones climáticas de tipo mediterráneo determinan un promedio de tan sólo veinte días de lluvia al año, que pueden descender a menos de diez durante la ocurrencia de años secos, de los cuales se presentan a lo menos tres cada diez años (ROMERO, 2004). Para que las aguas estén 
disponibles en las estaciones y años secos es fundamental que se almacenen en el suelo y subsuelo, lo que depende del proceso de infiltración gracias a suelos "no sellados", es decir con la porosidad y permeabilidad necesarias para permitir el almacenaje de las aguas en su interior (ROMERO, 2004).

Por otra parte, observamos que la teoría sostenibilista promueve que la conservación y restauración de los conectores ecológicos es una estrategia territorial imprescindible para conservar el capital natural de un país, debiendo ser una pieza en pleno diálogo con los sistemas urbanos y de infraestructuras. Al aportar grandes extensiones de habitats un conector ecológico hace las funciones de la matriz del paisaje. Esta convergencia entre las funciones de conector y de matriz es una característica altamente deseable (RODÁ, 2003), en la línea de considerar el conjunto del territorio y los papeles de las diversas piezas del conjunto. Muy estrechamente vinculados con la función conectora, se encuentran los ámbitos fluviales y costeros, que tienen además unos valores naturales, territoriales y sociales propios de primera magnitud.

Constatamos la pertinencia y oportunidad de la medida de reserva y prohibición de urbanizar el piedmont andino metropolitano junto a Santiago, y las partes altas de la cuenca en los planes en comento, además de prever espacios verdes relevantes a lo largo del Mapocho, y considerar como parques todos los cerros que rodean Santiago.

De igual manera, se dispuso en el plan gran diversidad y cantidad de parques al interior de la ciudad, destinados explícitamente no sólo a los fines de recreación sino también a fines de infiltración, además de sus otros valores como es el de generar islas de regulación del microclima de los barrios y vecindades (objetivo ya mencionado por Muñoz Maluschka desde los años 1930) (PAVEZ, 1993), como se verá a continuación.
3. Medidas para el incremento y diversificación funcional de las teselas verdes urbanas y su conexión entre ellas y con la matriz agrícola

Se sostiene que la heterogeneidad espacial es uno de los aspectos más relevantes de la estructura del paisaje y tiene gran influencia sobre su funcionamiento y también sobre los tipos y cantidades de bienes y servicios ecológicos que un territorio genera (RODÀ, 2003).

En los Planes de 1960 se abordó un sistema coordinado de espacios verdes de uso público, destinados al esparcimiento de la población y al saneamiento ambiental. En ellos no se podría ejecutar construcciones de ninguna naturaleza, salvo aquellas destinadas a complementar las funciones de esos espacios verdes como tales.

Se incorporaron los siguientes conceptos y reservas urbanas:

- Parques Intercomunales (polígonos): espacios verdes $\mathrm{y}$ otros destinados a reuniones juegos, deportes, y otros; distribuidos en cada cuadrante urbano, con superficies entre 30 y 100 hectáreas, contemplando un total de 852 hectáreas) (PARROCHIA et al., 1994).

- Parques Intercomunales Cuencas de Ríos: un total de 720 hectáreas al interior del límite intercomunal de la época.

- Parques Comunales: con una extensión que fluctúa entre 8 y 15 Hás., aprox. Se estableció un total de 354 hectáreas.

- Áreas Especiales: áreas verdes existentes de propiedad pública o privada, de uso controlado o restringido: Áreas Deportivas: Estadio Nacional, Club Hípico, Hipódromo, Estadio Colo-Colo, etc; Parques Privados: Parque Macul, Lo Hermida, Balneario Apoquindo, etc. 
- Áreas de Interés Histórico y Cultural: Convento Los Dominicos, en Apoquindo; Iglesia y Gruta de Lourdes, etc.

En materia de vialidad fueron propuestas “Avenidas Parques"(con una o dos calzadas para tránsito lento contiguas a una franja de área verde, con arboledas, jardines y juegos para niños, anchos entre 60 y $100 \mathrm{~m}$ ). También se consideró "Caminos Turísticos". Estas avenidas relacionaban los parques de diversas escalas.

El conjunto de políticas metropolitanas del Plan Intercomunal de Santiago aprobado desde 1960, debía servir de guía a los municipios y servicios estatales sectoriales, los que debían perfeccionarlas, sea a través de los Planes Reguladores Comunales y sus respectivos seccionales, o a través de Planes Seccionales específicos. Por otra parte, de igual forma, debían servir de guía para los planes coordinados equivalentes en las metrópolis de Valparaíso y Concepción.

Observamos que para la Región de Santiago de la época - con los asentamientos humanos en torno a las cuencas de los ríos Maipo-Mapocho y Aconcagua- se previó la formación de núcleos urbanos de diversos tamaños y especialidades, distribuidos de acuerdo a corredores de transporte y los recursos naturales de la región, con especial atención a la protección y desarrollo de los recursos agrícolas y a la ecología de la región en valles, montañas, y mares.

En el plano correspondiente al Plan Microregional de Santiago, con la desconcentración concentrada de Santiago propuesta -en su mayoría a partir de pueblos existentesse evitaría disgregar los espacios agrarios y forestales en miles de fragmentos cada viez más pequeños, menos funcionales y más desconectados. La naturaleza regional fue apreciada como un sistema dinámico y no como un escenario pasivo; se tenía conciencia que los servicios ecológicos serían prestados tanto por espacios singulares protegidos como por los sistemas agrarios, los cultivos extensivos de secano, los bosques y los matorrales.

A la luz de nuestra lectura del plan antes citado, constatamos que no se trataba en este plan de evitar simplemente que los espacios libres se incorporaran a los procesos de urbanización, ni de someterlos a una protección inmovilizante; se valoró estos espacios para dotarlos de un proyecto ecológico, social y económico propio, como única vía para garantizar su conservación en el largo plazo. Se tuvo conciencia de que el espacio urbano es una parte capital del territorio total, cuya solución no es posible sin una articulación integral urbano-rural. También constatamos el correcto manejo de las escalas en que se trabajaron los planos con los diversos proyectos de ordenamiento, dando cuenta de una clara comprensión de que ellas no aportan tanto, o tan sólo, la dimensión de las cosas, como "la naturaleza de los fenómenos"(FOLCH, 2003).

Con este enfoque, el plano correspondiente al Plan Micro-Regional de Santiago se reveló como una cartografía ambiental, donde no se prescinde del espacio urbano. Por el contrario, este se hace presente, y en él puede verse emergiendo las teselas verdes intercomunales existentes y proyectadas, y una continuidad entre la vialidad y ferrovías existentes y proyectadas en el área rural y aquellas existentes y proyectadas en el área urbana. El mantenimiento de corredores naturales en el interior de la ciudad (conectores ecológicos norte-sur y oriente poniente), y su interconexión con los refugios (teselas verdes urbanas mayores) y los escalones (teselas verdes urbanas menores) de creación humana, y de todos estos, a su vez, con la matriz geográfica, es un aspecto destacado en los Planes coordinados desde 1960, demostrando la temprana aplicación de la ecología del paisaje a la ciudad. Se expresa también los grandes equipamientos existentes y proyectados para el funcionamiento de los ámbitos rurales y urbanos de la región. 
Así, se fue intentando solucionar los efectos múltiples del proceso de metropolización de la ciudad de Santiago, la que desde 1960 comenzará a ser requerida en su pleno rol internacional. Su crecimiento basado en los recursos agua, energía y suelo, con las líneas de acción optimizadas y concurrentes: extensión por relleno, rehabilitación y remodelación, y satelización, respondía, de esta forma, a la preocupación que había estado presente en Chile desde los años 1950 , referida al eventual despliegue de un modelo de desarrollo físico "disperso"por efectos de la masificación del automóvil, y a la contaminación que ello acarrearía, entre otros efectos negativos (UNIVERSIDAD DE CHILE, 1958).

Se fijó una estructuración en bloques intercomunales autosuficientes, separados por cuñas verdes, una zonificación industrial, un sistema de multicentros cívicos, comerciales y de esparcimiento minimizando con ello la movilidad obligada, un sistema de áreas verdes públicas en diversas escalas y funciones, una red básica de transporte y vialidad en sus niveles regional, intercomunal y comunal. Esta última contempló nuevas carreteras de acceso a Santiago, y corredores de transporte norte-sur y oriente-poniente, confirmándose y perfeccionándose, además, un corredor de circunvalación de tres anillos propuesto desde 1936 (PAVEZ, 2006), todo lo cual vino a representar una verdadera refundación de la ciudad de Santiago.

El establecimiento de una banda suburbana, de transición entre lo urbano y lo rural, en base de parcelas y equipamientos metropolitanos, debía ser estable, dirigiéndose el crecimiento hacia el interior del área urbana o, en los diferentes satélites, más allá de su borde.

Así, sin impedir la expansión natural de la metrópolis, originada en sus recursos y sus relaciones geopolíticas internas y externas, se intentó lograr un conjunto de asentamientos urbanos a escala del hombre, en contacto permanente con la naturaleza, protegiendo los ecosistemas de la región y desarrollándolos armónicamente. Constatamos que una atención especial se prestó al tratamiento de los espacios fronterizos e intersticiales de los sistemas urbanos y peri-urbanos.

\section{Los cambios de prioridades guberna- mentales y los Planes Intercomunal y Microregional de Santiago 1960-1994}

Las políticas y conceptos contenidos en estos planes se cumplieron por 15 años, resultando un avance notable en la ordenación de la metrópoli. Ello, aun cuando ya las prioridades del gobierno de Frei Montalva (1964-70) se centraron, por una parte, en la remodelación urbana mediante megaproyectos residenciales ubicados al interior del pericentro metropolitano -perdiéndose la exigencia de coordinación de los planes intercomunales con los microregionales y regionales-, y por otra, en la idea de desconcentrar Santiago en la macroregión central de Chile, aunque en este último caso poco se avanzó.

En 2002 la metrópolis de Santiago (Gran Santiago) alcanzaba 5.300.000 habitantes, teniendo 100 veces más habitantes que la segunda ciudad de la región, Peñaflor, con 62.869 habitantes (GORE \& UNIVERSIDAD DE CHILE, 2002).

La Política Nacional de Desarrollo Urbano aplicada desde 1979, a causa de la opción del Gobierno del General Pinochet por el modelo de desarrollo económico y social neoliberal de manera radical, cambió la tendencia histórica, con la paulatina pérdida de las medidas de planificación para el ordenamiento territorial, y con un proceso de paulatino deconstructivismo en lo referido al patrimonio de espacios verdes urbanos y agrícolas, con el correspondiente impacto negativo en el paisaje, en la ecología urbano-regional y en la calidad de vida de sus habitantes. En el año 2002, cuando la población de la Región Metropolitana ya era de 6.038.974 habitantes, la cifra 
de espacios verdes era de sólo $3,3 \mathrm{~m}^{2} / \mathrm{hab}$. (GORE \& UNIVERSIDAD DE CHILE, 2002). El acceso a los ambientes naturales va siendo cada día más restringido, en un contexto en que las facilidades de transporte son mayores. La apertura de espacios privados a la recreación es limitada y produce constantes conflictos en áreas críticas como el piedmont andino metropolitano, privatizado ahora en toda su extensión (GORE et al., 2002). Se advierte que esto será aún más crítico a pesar que las superficies aptas para la recreación llegan a conformar casi el $70 \%$ de la superficie regional total que es de 1.534.900 hás. (GORE \& UNIVERSIDAD DE CHILE, 2002).

De los proyectos específicos que debían derivar de dichos planes, destacan los de vialidad y transporte (Primer Plan de Transporte, 1969, incluyendo el Metro de Santiago). Hubo, respecto de estos, una continuidad que sólo se vio interrumpida cuando el gobierno del Gral. Pinochet detuvo toda realización de obras públicas -incluido el Metro de Santiago- por 10 años a partir de 1975.

\section{CONCLUSIONES}

Vemos que, aun cuando se estaba lejos de contar con índices ecológicos con el fin de evaluar la vulnerabilidad del territorio frente a diferentes proyectos como son los de infraestructura, los principios fundamentales que se consideraron en los planes coordinados para Santiago desde 1960 dan cuenta de un temprano e inequívoco direccionamiento hacia la sustentabilidad.

Constatamos así que, aunque el objetivo de la sustentabilidad no es una novedad reciente para quienes fueron planificadores del espacio urbano-regional chileno del segundo y tercer cuarto del siglo XX, el paso a nuevas y mejores etapas del desarrollo se produce no solo lentamente entre nosotros, sino también con retrocesos.
Hoy la realidad de Santiago y su región es poco, o nada, sustentable en muchos aspectos, en un contexto que solo se interesa por el presente, negando el pasado y el futuro, pues desconoce las funciones y limitaciones del recurso espacio que se permite derrochar.

Parece necesario asumir, por otra parte, el carácter de discontinuidad y ruptura característico de nuestra realidad chilena y también latinoamericana, el que aflora cada cierto tiempo obligando a diseñar instrumentos de análisis que permitan comprender especialmente las sub-totalidades y fragmentos que se hacen presentes al momento de intervenir, y las señales que ellos aún emiten para su eventual incorporación a proyectos de futuro.

Finalmente, observamos en la actualidad que los tempranos aportes conceptuales del arquitecto y urbanista Muñoz Maluschka, reciclados y enriquecidos luego por sus discípulos, se adelantaron en mucho a su tiempo, reapareciendo varios de ellos como novedades recientes al comenzar el siglo XXI. Pensamos que la planificación urbana y regional con base geográfica, económica y social es una responsabilidad y un derecho social relevantes.

\section{BIBLIOGRAFÍA}

CHILE - MINISTERIO DE OBRAS PÚBLICAS, Dirección de Planeamiento, Departamento de Planes de Obras Públicas, (1962). BRIEVA ALVARADO, Amador y GONZÁLEZ MONTECINOS, Ventura. La Unidad Micro-Regional: características y métodos para delimitarla. Santiago de Chile, Publicación Nº6, junio de 1962 [Alvarado y González, autores en 1958].

CHILE - MINISTERIO DE OBRAS PÚBLICAS, Dirección de Planeamiento, Departamento Planos Reguladores, 1958, Plano Intercomunal de Santiago, "Anexo $\mathrm{N}^{\circ} 1$ : Funciones de la Dirección de Planeamiento del Ministerio de Obras Públicas", 
en: Documento-carpeta copia, nov. 1958, 20 págs. Archivo de J. Parrochia B.

FLORES S., E. 1966. "Sobre la cuenca de Santiago", en Anales de la Universidad de Chile, Año CXXIV, enero-marzo de 1966, $\mathrm{N}^{\circ} 137$, Santiago de Chile, pp. 65-87.

FOLCH, R. 2003. "Los conceptos socioecológicos de partida. Principios ecológicos versus criterios territoriales" (pp. 19-42), en: FOLCH, Ramón (coordinador), El territorio como sistema. Conceptos y herramientas de ordenación. Ed. Diputación de Barcelona, noviembre de 2003, 291 págs. ilustradas.

PESCI, R. 2003. "El urbanismo y la cultura ambiental. Sostenibilidad y levedad: territorio, urbanismo y ambiente" (pp. 101- 119) en: FOLCH, Ramón (coordinador). El territorio como sistema. Conceptos y herramientas de ordenación. Ed. Diputación de Barcelona, noviembre de 2003, 291 págs. ilustradas.

RODÀ, F. 2003. "La matriz del paisaje. Funciones ecológicas y territoriales" (pp. 43-55), en: FOLCH, Ramón (coordinador). El territorio como sistema. Conceptos y herramientas de ordenación. Ed. Diputación de Barcelona, noviembre de 2003, 291 págs. ilustradas.

GOBIERNO REGIONAL REGIÓN METROPOLITANA - UNIVERSIDAD DE CHILE. 2002. Criterios de Ordenamiento Territorial ambientalmente sustentable para la Región Metropolitana de Santiago. Propuesta. 41 páginas, octubre de 2002.

KRAUSE V., P. E. 1958. Macro-región de Santiago. Seminario de Investigación, Ciclo de Titulación de la Carrera de Arquitectura, Profs. Guías Arqtos. René Urbina, Fernando Kusnetzoff y Osvaldo Cáceres, Instituto de Vivienda, Urbanismo y Planeación, Facultad de Arquitectura de la Universidad de Chile, 1958, 130 págs., cuadros y gráficos.
LE CORBUSIER, 1971/73 [Publicación de 1942]. Principios del Urbanismo (La Carta de Atenas), Barcelona, Editorial Ariel, Esplugues de Llobregat, 1971/73.

[Nota: La Carta de Atenas es un manifiesto urbanístico redactado en el IV Congreso Internacional de Arquitectura Moderna (CIAM) celebrado a bordo del Patris II en 1933, en la ruta Marsella-Atenas, siendo publicado en 1942 por Le Corbusier].

PARROCHIA B., J.; PAVEZ R., M. I. 1994. El Plan Tridimensional de Ordenamiento Territorial y la Región Metropolitana de Santiago 1960-2000, Edición del Departamento de Urbanismo, Facultad de Arquitectura y Urbanismo de la Universidad de Chile, 216 págs. ilustradas, Santiago, 1994.

http://mazinger.sisib.uchile.cl/repositorio/ lb/arquitectura y urbanismo/pavezmi01/

PAVEZ R. M. I.; GUROVICH, A.; FERRANDO, F., HERNÁNDEZ, J. 20092010. El Corredor Fluvial del Río Mapocho Siglo XXI: hacia un conjunto de variables para la definición y el manejo de un polígono de intervención multifacética e integral entre Quinta Normal y El Monte. Proyecto FONDECYT 1090199, 2009-2010.

http://ri.conicyt.cl/575/article-33416.html

PAVEZ R., M. I. 2006. Vialidad y transporte en la metrópoli de Santiago 1950-1979: concepto y estrategia de ordenación del territorio en el marco de la Planificación Urbana y Regional por el Estado de Chile. Tesis Doctoral, Director Dr. Julio Pozueta E., ETSA, Universidad Politécnica de Madrid, 13 nov. 2006.

http://oa.upm.es/452/

http://captura.uchile.cl/dspace/ handle/2250/5098 
PAVEZ R., M. I. 2003. En la ruta de Juan Parrochia Beguin - Premio Nacional de Urbanismo Chile 1996. FACULTAD DE ARQUITECTURA Y URBANISMO - VICERRECTORÍA DE INVESTIGACIÓN Y DESARROLLO U. Chile, Santiago, ISBN Nº56-19-0407-1, julio 2003, 319 págs., 465 ilustraciones.

PAVEZ R., M. I. 1993. Luis Muñoz Maluschka: Escritos. Ed. D. Urbanismo, F.A.U. U.CH., nov. 1993, 86 págs. ilustradas.
ROMERO A., H. 2004. "Crecimiento espacial de Santiago entre 1989 y 2003 y la pérdida de servicios ambientales". En: TUPPER, Patricio, Editor. 2004, Hacer ciudad. (Centro Chileno de Urbanismo - Agrupación Defendamos la Ciudad), pp. 179-201.

UNIVERSIDAD DE CHILE, Departamento de Extensión Cultural, 1958. BOLETIN INFORMATIVO $N^{\circ} 34,2^{a}$ y $3^{a}$ etapa del Seminario del Gran Santiago, Santiago, Chile, octubre 1958. 